\title{
Management of Myasthenia Gravis
}

\author{
Sudhir Venkataramaiah ${ }^{1}$ Sriganesh Kamath ${ }^{1}$ \\ ${ }^{1}$ Department of Neuroanaesthesiology and Neurocritical Care, \\ National Institute of Mental Health and Neurosciences, Hosur \\ Road, Bengaluru, Karnataka, India
}

\begin{abstract}
Address for correspondence Sudhir Venkataramaiah, MD, Department of Neuroanaesthesiology and Neurocritical Care, National Institute of Mental Health and Neurosciences, Hosur Road, Bengaluru-560029, Karnataka, India (e-mail: vsudhir77@gmail.com).
\end{abstract}

\begin{abstract}
Keywords

- acetyl choline receptor

- anticholinesterase

- immunoglobulin

- myasthenia gravis

- neuromuscular junction

- plasmapheresis

Myasthenia gravis (MG) apart from Guillain-Barré syndrome is one of the most common disorders presenting as respiratory failure secondary to muscle weakness in the modern world. MG is also one of the most researched and reasonably well understood autoimmune disorder of mankind. From the description of early cases of MG to the current day understanding, the progress in the management and therapeutics has advanced significantly. Diagnosis of MG can be accurately done either with traditional tests such as the edrophonium test or by advanced nerve conduction studies. Presence of demonstrable circulating autoantibodies against the acetyl choline receptors, muscle-specific tyrosine kinase, involvement of $B$ and $T$ cells in the pathogenesis, and tomographic evidence of the enlarged thymus gland in certain patients have led to designing specific treatment strategies. Removal of the circulating autoantibodies as much as possible by plasmapheresis supported by anticholinesterases and immunosuppression are the mainstay of targeted therapy. The advent of newer immunosuppressant drugs such as rituximab which targets the CD20 protein present on the surface of B cells and tacrolimus which is an interleukin 2 inhibitor has improved the therapeutic armamentarium of the physician. These agents in combination with time tested medications such as steroids and antimetabolites have rendered faster remission rates and improved outcomes in MG crisis. This article outlines the basic pathophysiology, specific and supportive management strategies in patients presenting with MG with or without crisis.
\end{abstract}

\section{Introduction}

Myasthenia gravis (MG) is characterized by fluctuating muscular weakness, which is relieved by cessation of activity and aggravated by intense physical activity. Majority of the patients are adults; however, an increased incidence in children below 15 years of age has been reported in certain Asian regions. ${ }^{1}$ The presentation initially may be limited to the extraocular muscles termed as "ocular myasthenia gravis" which may progress to involve bulbar and skeletal muscles to manifest as generalized MG.

\section{Pathophysiology}

Myasthenia gravis is essentially the disease of the neuromuscular junction. The neuromuscular junction anatomically is

received

February 23, 2019 accepted after revision

April 1, 2019

published online

June 7, 2019 composed of the presynaptic, synaptic, and post-synaptic components. Acetyl choline (ACh) is synthesized, stored in the presynaptic nerve terminal, and released into the synaptic clefts in quanta secondary to the actions of calcium when an impulse arrives at the nerve terminal $(\boldsymbol{-}$ Fig. $\mathbf{1}$ ). The acetylcholinesterase enzyme is present in these clefts that hydrolyzes the ACh to terminate the transmission. The ACh inhibiting agents act here in improving the clinical symptoms. The post-synaptic membrane contains the ACh receptors (AChRs), the detailed anatomy of which can be found elsewhere. ${ }^{2}$ The most common form of MG, seropositive MG, features autoantibodies ( $\boldsymbol{- \text { Fig. }}$ 2) which accelerate the endocytosis of the receptor or induce complement-mediated destruction of the AChR. ${ }^{3}$ About 10 to $20 \%$ of the MG patients do not demonstrate autoantibodies against the AChRthis condition is called "seronegative MG." These patients

Copyright $\odot 2019$ Indian Society of Neuroanaesthesiology and Critical Care
License terms

(a) (1) $\ominus \circledast$ 


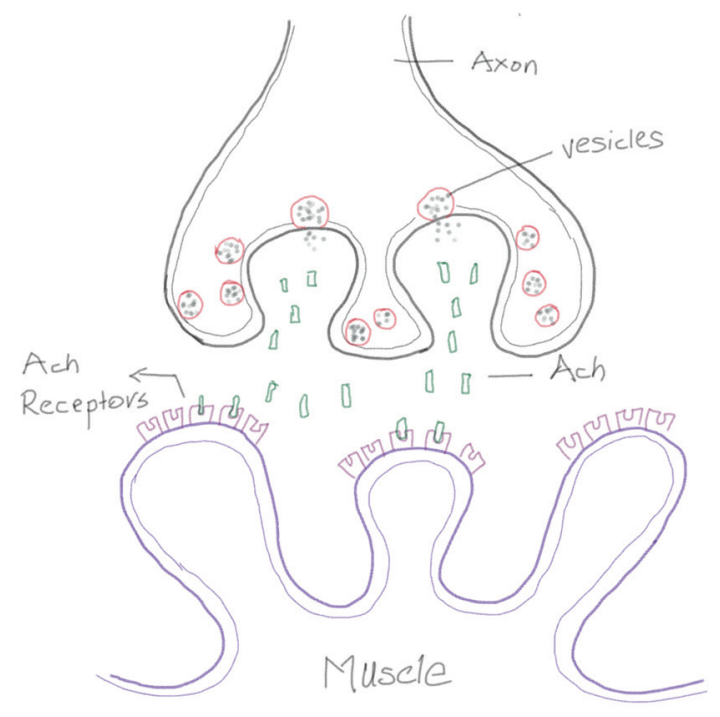

Fig. 1 The neuromuscular junction in a healthy individual.

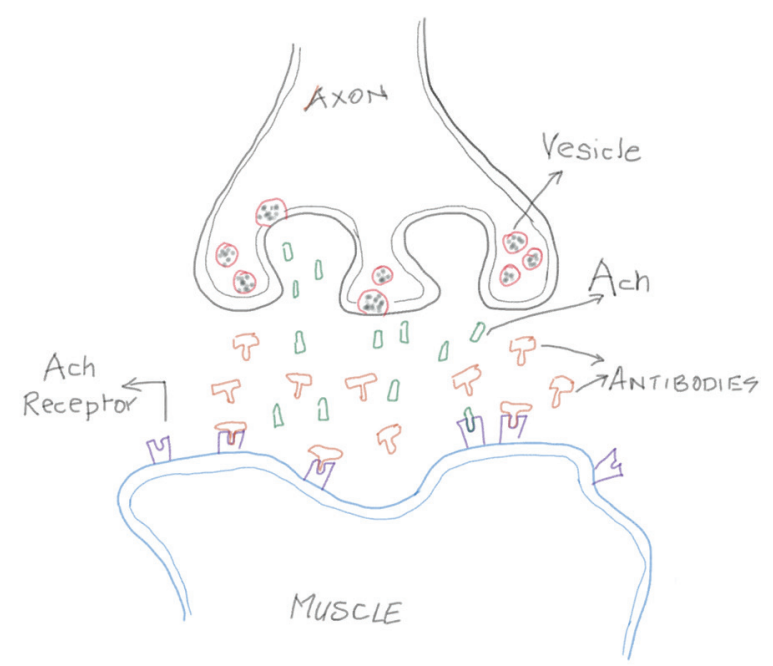

Fig. 2 The neuromuscular junction in an individual suffering from myasthenia gravis.

demonstrate antibodies directed toward a receptor tyrosine kinase called muscle-specific kinase (MuSK). MuSKs are required to cluster the AChRs in groups and their production is mediated by a protein in the basement membrane called Agrin. The exact means by which Agrin activates the MuSK is not clearly understood. ${ }^{4,5}$ Though the antibodies against AChRs are produced by B cells, $\mathrm{T}$ cells also have a role in this autoimmune response. $T$ cells, specifically the CD4+ helper cells, aid in the production and activation of the proliferation of $B$ cells which produce immunoglobulins against AChRs. ${ }^{6,7}$

The thymus gland also plays an essential role in the pathogenesis of MG, especially in those patients who present with thymomas. Myoid cells which are present in the medullary region of the thymus along with the $\mathrm{T}$ helper cells produce antibodies against AChRs. Any alterations in the body physiology from either genetic or viral disease lead to tolerance induction to self-antigens and responsiveness of the lymphocytes to alien antigens. ${ }^{8,9}$

\section{Clinical Classification of Myasthenia Gravis}

Improvising on the original Osserman's classification of MG, the American Myasthenia Gravis Foundation has proposed a more comprehensive classification for the purpose of standardization. ${ }^{8}$ The classification is as follows:

1. Any ocular weakness, strength of all other muscles being normal.

2. Mild weakness other than ocular muscles, $+/ 2$ weakness of ocular muscles of any severity. 2a: Predominant limb and/ or axial involvement, $2 \mathrm{~b}$ : predominant oropharyngeal and/or respiratory involvement.

3. Moderate weakness affecting muscles other than ocular muscles, may have ocular weakness. 3a: Predominant limb and/or axial involvement; 3b: predominant oropharyngeal and/or respiratory involvement.

4. Severe weakness affecting muscles other than ocular muscles, may have ocular weakness. 4a: Predominant limb and/or axial involvement; $4 \mathrm{~b}$ : predominant oropharyngeal and/or respiratory involvement.

5. Defined by intubation with or without mechanical ventilation, except when employed during routine postoperative management. The use of feeding tube without intubation places the patient in class $4 \mathrm{~b}$.

\section{Clinical Features and Diagnosis of Myasthenia Gravis}

Presence of shifting weakness of skeletal muscle groups, which aggravates by physical activity and improves on resting, is the hallmark of MG. In most of the cases, the weakness is initially observed in the ocular muscles which then spreads on to bulbar and skeletal muscles. Respiratory muscles are involved in the end which necessitates the intensive care management of the patient.

A few tests will confirm the diagnosis of MG. The most widely used test is the edrophonium/Tensilon test. Under close monitoring, the patient is injected with edrophonium-a short acting acetylcholinesterase inhibitor. An objective improvement in the muscle strength primarily the ocular muscle indicates a positive test. ${ }^{10}$ An ice pack placed on the eye on the other hand improves the ptosis which is referred to as the "ice pack" test. The ice pack test is employed in those patients where edrophonium test is not indicated. ${ }^{11}$ Electrophysiological tests can be performed for the diagnosis of MG. Single fiber electromyography is considered to be the most effective test where action potentials of individual nerve fibers can be identified. A delay in the second action potential following the first indicates a positive test. ${ }^{10}$ The other neurophysiological test used is the repetitive nerve stimulation study where a nerve is repeatedly stimulated supramaximally, and a 
$10 \%$ decrement between the first and the fifth stimuli is diagnostic of MG. ${ }^{12}$ In all patients with confirmed MG, a computed tomography scan of the thorax has to be done to look for thymoma.

\section{Evaluation and Supportive Management of Myasthenia Gravis}

Majority of the patients with MG without the involvement of respiratory muscles are managed in the ward or as outpatients with specific therapies, detailed below. Weakness of respiratory muscles and bulbar involvement require close monitoring, intensive care, and ventilatory assistance. Inability to clear oral and bronchial secretions; drooling of saliva; nasal voice; or choking on oral secretions indicates a severe bulbar involvement in which case tracheal intubation is imminent for airway protection.

On clinical examination, a patient in acute crisis will present with tachypnea, tachycardia, sweating, accessory muscle involvement to sustain ventilation, paradoxical breathing, drooping of the neck which indicates intense diaphragm fatigue and staccato speech (unable to complete speaking a whole sentence in one breath). ${ }^{13}$ Bedside pulmonary function tests and spirometry can be performed in clinically stable and co-operative patients in sitting position. Single breath count test and neck flexor strength have been shown to have good correlation with spirometry in predicting reduced forced vital capacity (FVC). ${ }^{14} \mathrm{~A}$ normal individual can count up to 50 counts after a single deep inspiration. A count of up to 25 approximately correlates with FVC of $2 \mathrm{~L}$ and a count less than 15 indicates significantly less respiratory reserve. ${ }^{15}$ An arterial blood gas (ABG) analysis will indicate the severity of ventilatory failure and $\mathrm{CO}_{2}$ accumulation. However, the ABG has to be interpreted with great caution since compensatory changes occur, and $\mathrm{CO}_{2}$ levels may be normal in a tachypneic patient.

If the clinical condition of the patient is stable with mild-to-moderate weakness and not in overt respiratory failure, then the patient is to be managed medically with immunosuppressive, anticholinergic, and other indicated medications under close monitoring in the ward/high dependency unit. If the patient is demonstrating severe muscle weakness with bulbar involvement, and/or overt respiratory failure with hypercapnia, the risk of pulmonary aspiration is imminent or may have already occurred. Such a patient must be immediately intubated for airway protection and ventilatory management in the intensive care unit (ICU). In case the patient is in mild respiratory distress, with no bulbar involvement, conscious and oriented, not overtly hypercapnic, these patients can be given a trial of noninvasive ventilation (CPAP/BiPAP; continuous positive airway pressure/bi-level positive air way pressure) with frequent clearing of the oral cavity by suctioning. BiPAP has been shown to reduce both ICU and hospital stay even in patients presenting with myasthenic crisis. ${ }^{16}$ A preliminary trial showed that BiPAP may prevent tracheal intubation in patients who present with myasthenic crisis and are not overtly hypercapnic. ${ }^{17}$

\section{Intensive Care Management of Patients with Myasthenia Gravis}

The decision to intubate and mechanically ventilate the patient should be based on the clinical judgement of the treating physician. More than the results of the ABG, bedside respiratory reserve measurements and vital parameters (pulse rate and $\mathrm{BP}$ ) take precedence. To keep matters simple, a 20/30/40 rule (FVC $<20 \mathrm{~mL} / \mathrm{kg}$; negative inspiratory pressure [NIP] $<30 \mathrm{~cm} \mathrm{H}_{2} \mathrm{O}$; and positive expiratory pressure [PEP] $<40 \mathrm{~cm} \mathrm{H}_{2} \mathrm{O}$ ) can guide the decision to intubate. ${ }^{18}$ Duration of mechanical ventilation is unpredictable in MG. Release from mechanical ventilation and extubation depends on how rapidly the plasma can be cleared of autoantibodies, and the response to anticholinergic medications and immune suppressants. Meanwhile, the patient is to be managed according to the general principles of critical care-respiratory care, addressing infections, and attention to nutrition along with the targeted therapy. Early versus delayed tracheostomy is still a controversy with no clear guidelines available at present.

Clinical improvement in muscle power can be taken as a guide to initiate weaning. An FVC $>15 \mathrm{~mL} / \mathrm{kg}$, NIP $>20 \mathrm{~mL} / \mathrm{kg}$, and a PEP $>40 \mathrm{~mL} / \mathrm{kg}$ indicate a possible successful weaning process. ${ }^{18,19}$ Similarly, a patient demonstrating an FVC $>25$ $\mathrm{mL} / \mathrm{kg}, \mathrm{NIP}>40 \mathrm{~mL} / \mathrm{kg}$, and PEP $>50 \mathrm{~mL} / \mathrm{kg}$ may be considered a suitable candidate for extubation. Clinically, a good cough reflex and improvement in the neck flexor power facilitating a sustained neck lift indicate good resolution in weakness of the bulbar muscles, and extubation in these patients may be successful. ${ }^{20}$

Atelectasis, decreased FVC, and acidosis are important predictors of reintubation. ${ }^{21}$ About one-fourth of the extubated patients get reintubated. ${ }^{21,22}$ Reintubated patients tend to spend a longer time in the ICU. Early decision to intubate and tracheostomy, high-quality chest physiotherapy, frequent change in positioning, and early mobilization from the bed during off ventilator hours can aid in preventing reintubation. ${ }^{23}$

Occasionally, we come across patients who do not respond to plasmapheresis or anticholinesterases and weakness continues to persist. Steroid-induced myopathy is a possibility in such patients. Gradual tapering and cessation of steroids will improve the clinical condition. ${ }^{24,25}$

\section{Specific Management of Myasthenia Gravis}

Several specific treatments are available for the management of MG. However, significant variation in the treatment is noted because good-quality randomized controlled trials of MG therapies are rare and generalizability of the available evidence is difficult due to the heterogeneous nature of the disease. Hence, experts and societies have developed treatment statements and clinical practice guidelines by consensus to guide clinicians on multipronged approach to MG management. ${ }^{26,27}$ The limitations of treatment statements are that the recommendation depends on the constituents of the expert panel and also needs updating every few years with availability of new evidence. ${ }^{28}$ 
Following are the classes of specific treatment options for medical management of MG.

1. Acetylcholinesterase inhibitors (pyridostigmine and neostigmine)

2. Corticosteroids (prednisolone)

3. Plasma exchange (PLEX)

4. Intravenous immunoglobulin (IVIG)

5. Immunosuppressants (azathioprine, rituximab, cyclosporine, mycophenolate mofetil, methotrexate, tacrolimus)

\section{Acetylcholinesterase Inhibitors}

As a first-line therapy for mild MG, acetylcholinesterase inhibitors slow down the degradation of acetylcholine and prolong their effect on the neuromuscular junction providing symptom benefits. Pyridostigmine administered orally has a rapid onset of action ( $<30$ minutes) and the action lasts for 3 to 4 hours. The effective dose ranges from 30 to $90 \mathrm{mg}$ every 4 to 6 hours. Neostigmine is used intramuscularly along with anticholinergic drugs to reduce its side effects (excessive secretions, bradycardia) as 0.5 to $1 \mathrm{mg}$ every 4 to 6 hours under close monitoring. When treatment with these drugs alone fails, immunosuppressant drugs are considered. ${ }^{29}$

\section{Corticosteroids}

Prednisone is the most commonly used drug which leads to significant improvement in three-fourth of patients with ocular or generalized MG. The effective dose depends on the severity of symptoms. Patients with mild-to-moderate symptoms need approximately 20 to $40 \mathrm{mg} /$ day of drug while those with severe symptoms may require up to 1 $\mathrm{mg} / \mathrm{kg} /$ day. During the initial 1 to 2 weeks, approximately $50 \%$ of patients may develop worsening of weakness. Therefore, high-dose regimen is initiated in the hospital along with PLEX or IVIG. Otherwise, outpatient dose-escalation strategy is followed till target dose is achieved over several weeks. Tapering of the dose is initiated after a significant improvement in symptoms is established for 1 to 2 months. Tapering by 5 to $10 \mathrm{mg} / \mathrm{month}$ is preferred, with slower withdrawal once $30 \mathrm{mg} /$ day dose is reached. The maintenance dose thereafter is approximately $5 \mathrm{mg} /$ day. Side effects such as weight gain, cushingoid features, easy bruising, cataracts, glaucoma, hypertension, diabetes, dyslipidemia, and osteoporosis should be closely monitored. Calcium (1.5 g/day) and vitamin D (400-800 IU/day) should be supplemented and those at risk of osteoporosis should also receive a bisphosphonate. Other immunosuppressants, which have delayed onset of action, should be added early in the treatment to either replace or reduce corticosteroid dose and reduce its long-term adverse effects. ${ }^{30}$

\section{Rapid Onset Immune Therapies}

Rapid-onset immune therapies such as IVIG and PLEX ( - Table 1) are first-line therapies for worsening MG or myasthenic crisis, described as respiratory failure from bulbar or diaphragmatic muscle weakness mostly provoked by infection, surgery, or medications. Rapid treatment effects make them useful as a short-term rescue therapy for rapidly worsening symptoms or as a preparation for surgery. IVIG and PLEX are equally effective for the treatment of moderate-to-severe MG with regard to functional outcomes and quality of life scores at 14 and 28 days. ${ }^{31}$ The choice between PLEX and IVIG is, therefore, based on the mechanism of action, efficacy, adverse effects, cost, and availability. PLEX is preferred in myasthenic crisis as it has a relatively faster onset of action then IVIG. However, if expertise is lacking, IVIG should be used. For disabling but nonlife-threatening symptoms, IVIG may be preferred. ${ }^{32}$

\section{Intravenous Immunoglobulin}

Intravenous Immunoglobulin is obtained from pooled blood donors and contains purified immunoglobulin $G(\operatorname{IgG})$. The exact mechanism by which it acts is unclear but likely involves blockade of Fc receptors on macrophages, reduced complement activation, and reduced production of antibodies and cytokines. The usual dose of IVIG is $2 \mathrm{~g} / \mathrm{kg}(0.4 \mathrm{~g} / \mathrm{kg} /$ day) over 2 to 5 days. Improvement in symptoms is observed by 1 week and the effect lasts for several weeks in more than $50 \%$ of patients. Common side effects of IVIG include chills, fever, headache, and serious, rarer side effects include stroke, leukopenia, renal dysfunction, and aseptic meningitis. ${ }^{32}$ Presence of cardiac disease may warrant IVIG whereas IVIG may not be appropriate in renal dysfunction.

\section{Plasma Exchange}

Plasma Exchange involves apheresis where circulating immunoglobulins, complement, immune complexes, cytokines and other inflammatory mediators are removed. The normal course involves exchange of approximately 1 to 1.5 times the blood volume, performed on alternate days over five to six sittings. Daily small volume PLEX is performed in those with expected large fluid shifts. Improvement in symptoms is observed in majority of patients (especially MuSK-antibody positive MG) within few days and lasts for approximately 3 months. Adverse effects related to PLEX include paresthesias (citrate-induced hypocalcemia), hypotension (from large fluid shifts), arrhythmias, nausea, chills, hypoproteinemia, dyselectrolytemia, and peripheral edema. Complications from central venous catheter (CVC) for PLEX include thrombosis, bacteremia, pneumothorax, and thrombophlebitis.32 Literature reports PLEX to be costlier than IVIG, largely from the need for increased staff, CVC placement and care, longer treatment course, and management of complications. However, in India, PLEX remains a significantly cheaper option than IVIG.

\section{Azathioprine}

The effect of azathioprine begins 6 to 12 months after initiation and reaches a peak after 1 to 2 years. More than $90 \%$ of patients improve with azathioprine alone or with con-

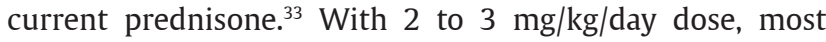
patients can be weaned off prednisolone and yet maintain remission for few years. The common side effects include nausea, vomiting, and malaise, while the less common side effects are hematologic and liver function abnormalities and pancreatitis. Monthly monitoring of blood cell counts and liver function during the initial 6 months and less frequently 
during later stages is desirable. Patients with thiopurine methyltransferase gene deficiency ( 1 in 300 people) should not receive azathioprine in view of life-threatening bone marrow suppression. ${ }^{34}$

\section{Mycophenolate Mofetil}

There is contrasting evidence for the efficacy of mycophenolate mofetil in MG. However, this drug is well tolerated with a few side effects. At 1 to $3 \mathrm{~g} /$ day, significant improvement in symptoms and remission with steroid dose reduction was noted. ${ }^{35}$ However, a later trial failed to demonstrate beneficial effect of $2 \mathrm{~g} /$ day mycophenolate when administered for 36 weeks. ${ }^{36}$ Common side effects include nausea, diarrhea, leukopenia, and infections necessitating frequent blood counts monitoring during the therapy. Major fetal malformations are reported with mycophenolate use during pregnancy. ${ }^{30}$

\section{Cyclosporine}

Cyclosporine has relatively faster onset of action (1-2 months) among nonsteroidal immunosuppressant drugs. A $5 \mathrm{mg} / \mathrm{kg} /$ day dose with 100 to $150 \mathrm{ng} / \mathrm{mL}$ serum level is generally recommended. Due to significant renal, hepatic, and hematologic toxicity and major drug interactions, its use is decreasing. ${ }^{30}$

\section{Methotrexate}

Methotrexate is a selective inhibitor of dihydrofolate, ${ }^{29}$ and its steroid-sparing effect is reported to be similar to that of azathioprine. ${ }^{37}$ However, a recent RCT found no steroid sparing benefit with oral methotrexate $20 \mathrm{mg} /$ week in patients with generalized MG during the 12 -month study period. ${ }^{38}$

\section{Rituximab}

Rituximab is a monoclonal antibody against B cell membrane marker CD20. It is found to be efficacious in generalized MG refractory to other immunosuppressants, ${ }^{39}$ especially for MuSK antibody-positive disease. The standard dose is 375 $\mathrm{mg} / \mathrm{m}^{2} /$ week for 4 consecutive weeks. Common side effects include depletion in peripheral B cells (within 2 weeks), flushing, and chills (with first dose) and susceptibility for infections. ${ }^{30}$

\section{Cyclophosphamide}

Cyclophosphamide is an alkylating agent that reduces B and $\mathrm{T}$ cell proliferation. The commonly used regimen is six cycles of intravenous cyclophosphamide $\left(0.75 \mathrm{~g} / \mathrm{m}^{2}\right)$ every 4 weeks followed by oral immunosuppression. ${ }^{40}$ Though effective, potentially serious side effects limit its use to patients who are refractory to other immunotherapies. ${ }^{30}$

\section{Tacrolimus}

Tacrolimus is a macrolide immunosuppressant which inhibits the production of interleukin-2. The dose used for MG along with conventional treatment is 3 to $5 \mathrm{mg} /$ day $(0.1 \mathrm{mg} /$ $\mathrm{kg} /$ day) for 6 to 36 months. The adverse effects are mild and most commonly include increase in hemoglobin A1C level and neutrophil count and transient alterations in renal and liver function tests. Tacrolimus with adequate treatment duration might have steroid-sparing effect in the management of MG without major side effects. ${ }^{41}$

\section{Leflunomide}

Leflunomide is an immunosuppressant that blocks pyrimidine nucleotide biosynthesis. A pilot study in 15 patients demonstrated good tolerance and efficacy with leflunomide $20 \mathrm{mg}$ for corticosteroid-dependent MG which resulted in significant lowering of corticosteroid dose by 6 months. ${ }^{42}$

\section{Surgical Management: Thymectomy}

Thymectomy should be considered for patients with thymoma. If thymectomy is not possible, chemo-radiotherapy may be considered to relieve symptoms and prevent local invasion. For patients without thymoma, thymectomy may be beneficial in younger ( $<60$ years) patients with generalized MG. Medication-free remission and symptom-free state are high after thymectomy, though this may take several years. ${ }^{43}$

The optimal timing of thymectomy is not clear; however, thymectomy is recommended during the initial 3 years of diagnosis. ${ }^{44}$ The response rate from thymectomy is similar for both AChR antibody-positive and seronegative patients.

\section{New Drugs Undergoing Evaluation}

Rozanolixizumab (UCB7665) is an antihuman neonatal Fc receptor monoclonal antibody aimed at reducing IgG levels. It is currently in phase II trial for MG treatment.

Efgartigimod (ARGX-113), Fc receptor monoclonal antibody, demonstrated clinical improvement in $75 \%$ of patients with MG over a 6-week study period compared with $25 \%$ improvement in placebo in the recently concluded phase II trial with reduction in IgG levels and good tolerance. Monarsen (EN101) is an antisense oligonucleotide which reduces the production of acetylcholinesterase enzyme. ${ }^{45}$

\section{Conclusion}

Though mortality from myasthenic crisis has become very rare, morbidity still exists. Better understanding of the pathophysiology and advancement in therapeutic interventions

Table 1 Immune-modulating drugs for MG based on timing of onset and duration of action

\begin{tabular}{|l|l|l|}
\hline Onset of action & $\begin{array}{l}\text { Duration of } \\
\text { action }\end{array}$ & Drugs \\
\hline Fast & $\begin{array}{l}\text { Short (days to } \\
\text { weeks) }\end{array}$ & PLEX, IVIG \\
\hline Intermediate & $\begin{array}{l}\text { Intermedi- } \\
\text { ate (weeks to } \\
\text { months) }\end{array}$ & $\begin{array}{l}\text { Corticosteroids, } \\
\text { cyclosporine, cy- } \\
\text { clophosphamide, } \\
\text { methotrexate }\end{array}$ \\
\hline Slow & $\begin{array}{l}\text { Prolonged } \\
\text { (months to years) }\end{array}$ & $\begin{array}{l}\text { Thymectomy, } \\
\text { azathioprine, } \\
\text { rituximab, } \\
\text { mycophenolate }\end{array}$ \\
\hline
\end{tabular}

Abbreviations: IVIG, intravenous immunoglobulin; MG, myasthenia gravis; PLEX, plasma exchange. 
have further been able to contain the morbidity. Even then the time to clinically significant remission of the disease is variable. In the intensive care setting, aggressive pulmonary care, good nutritional support, early intubation/tracheostomy, judicious weaning, and extubation decisions along with specific targeted therapy will improve the outcomes and reduce hospital stay in MG patients.

Conflict of Interest

None declared.

\section{References}

1 Zhang X, Yang M, Xu J, et al. Clinical and serological study of myasthenia gravis in HuBei Province, China. J Neurol Neurosurg Psychiatry 2007;78(4):386-390

2 Slater CR. The structure of human neuromuscular junctions: some unanswered molecular questions. Int J Mol Sci 2017;18(10):E2183

3 Drachman DB, Adams RN, Stanley EF, Pestronk A. Mechanisms of acetylcholine receptor loss in myasthenia gravis. J Neurol Neurosurg Psychiatry 1980;43(7):601-610

4 Wallace B. scFvs get down to basics: how MuSK makes synapses. Nat Biotechnol 1997;15(8):721-722

5 Wickelgren I. Synapse-making molecules revealed. Science 1996;272(5265):1100

6 Drachman DB, McIntosh KR, Yang B. Factors that determine the severity of experimental myasthenia gravis. Ann N Y Acad Sci 1998;841:262-282

7 Hughes BW, Moro De Casillas ML, Kaminski HJ. Pathophysiology of myasthenia gravis. Semin Neurol 2004;24(1):21-30

8 Thanvi BR, Lo TC. Update on myasthenia gravis. Postgrad Med J 2004;80(950):690-700

9 Vincent A, Palace J, Hilton-Jones D. Myasthenia gravis. Lancet 2001;357(9274):2122-2128

10 Jayam Trouth A, Dabi A, Solieman N, Kurukumbi M, Kalyanam J. Myasthenia gravis: a review. Autoimmune Dis 2012;2012:874680

11 Cheo SW, Low QJ, Mow WC, Yuen Kang C. Ice pack test - an useful bedside test to diagnose myasthenia gravis. QJM 2018.

12 Sanders DB, Howard JF Jr, Johns TR. Single-fiber electromyography in myasthenia gravis. Neurology 1979;29(1):68-76

13 Rabinstein AA. Acute Neuromuscular Respiratory Failure. Continuum (Minneap Minn). 2015;21(5 Neurocritical Care):1324-1345

14 Elsheikh B, Arnold WD, Gharibshahi S, Reynolds J, Freimer M, Kissel JT. Correlation of single-breath count test and neck flexor muscle strength with spirometry in myasthenia gravis. Muscle Nerve 2016;53(1):134-136

15 Rezania K, Goldenberg FD, White S. Neuromuscular disorders and acute respiratory failure: diagnosis and management. Neurol Clin 2012;30(1):161-185, viii

16 Seneviratne J, Mandrekar J, Wijdicks EF, Rabinstein AA. Noninvasive ventilation in myasthenic crisis. Arch Neurol 2008;65(1):54-58

17 Rabinstein A, Wijdicks EF. BiPAP in acute respiratory failure due to myasthenic crisis may prevent intubation. Neurology 2002;59(10):1647-1649

18 Godoy DA, Mello LJ, Masotti L, Di Napoli M. The myasthenic patient in crisis: an update of the management in neurointensive care unit. Arq Neuropsiquiatr 2013;71(9A):627-639

19 Kirmani JF, Yahia AM, Qureshi AI. Myasthenic crisis. Curr Treat Options Neurol 2004;6(1):3-15

20 Wendell LC, Levine JM. Myasthenic crisis. Neurohospitalist 2011;1(1):16-22
21 Seneviratne J, Mandrekar J, Wijdicks EF, Rabinstein A. Predictors of extubation failure in myasthenic crisis. Arch Neurol 2008;65(7):929-933

22 Rabinstein AA, Mueller-Kronast N. Risk of extubation failure in patients with myasthenic crisis. Neurocrit Care 2005;3(3):213-215

23 Varelas PN, Chua HC, Natterman J, et al. Ventilatory care in myasthenia gravis crisis: assessing the baseline adverse event rate. Crit Care Med 2002;30(12):2663-2668

24 Panegyres PK, Squier M, Mills KR, Newsom-Davis J. Acute myopathy associated with large parenteral dose of corticosteroid in myasthenia gravis. J Neurol Neurosurg Psychiatry 1993;56(6):702-704

25 Vallet B, Fourrier F, Hurtevent JF, Parent M, Chopin C. Myasthenia gravis and steroid-induced myopathy of the respiratory muscles. Intensive Care Med 1992;18(7):424-426

26 Sussman J, Farrugia ME, Maddison P, Hill M, Leite MI, Hilton-Jones D. The Association of British Neurologists' myasthenia gravis guidelines. Ann N Y Acad Sci 2018;1412(1):166-169

27 Sanders DB, Wolfe GI, Benatar M, et al. International consensus guidance for management of myasthenia gravis: executive summary. Neurology 2016;87(4):419-425

28 Sanders DB, Wolfe GI, Narayanaswami P; MGFA Task Force on MG Treatment Guidance. Developing treatment guidelines for myasthenia gravis. Ann N Y Acad Sci 2018;1412(1):95-101

29 Jones LA, Robertson NP. An update on treatments in myasthenia gravis. J Neurol 2017;264(1):205-207

30 Li Y, Arora Y, Levin K. Myasthenia gravis: newer therapies offer sustained improvement. Cleve Clin JMed 2013;80(11):711-721

31 Barth D, Nabavi Nouri M, Ng E, Nwe P, Bril V. Comparison of IVIg and PLEX in patients with myasthenia gravis. Neurology 2011;76(23):2017-2023

32 Dhawan PS, Goodman BP, Harper CM, et al. IVIG versus PLEX in the treatment of worsening myasthenia gravis: what is the evidence?: a critically appraised topic. Neurologist 2015; 19(5):145-148

33 Mertens HG, Hertel G, Reuther P, Ricker K. Effect of immunosuppressive drugs (azathioprine). Ann N Y Acad Sci 1981;377:691-699

34 Relling MV, Gardner EE, Sandborn WJ, et al; Clinical Pharmacogenetics Implementation Consortium. Clinical Pharmacogenetics Implementation Consortium guidelines for thiopurine methyltransferase genotype and thiopurine dosing. Clin Pharmacol Ther 2011;89(3):387-391

35 Meriggioli MN, Ciafaloni E, Al-Hayk KA, et al. Mycophenolate mofetil for myasthenia gravis: an analysis of efficacy, safety, and tolerability. Neurology 2003;61(10):1438-1440

36 Sanders DB, Hart IK, Mantegazza R, et al. An international, phase III, randomized trial of mycophenolate mofetil in myasthenia gravis. Neurology 2008;71(6):400-406

37 Heckmann JM, Rawoot A, Bateman K, Renison R, Badri M. A single-blinded trial of methotrexate versus azathioprine as steroid-sparing agents in generalized myasthenia gravis. BMC Neurol 2011;11:97

38 Pasnoor M, He J, Herbelin L, et al; Methotrexate in MG Investigators of the Muscle Study Group. A randomized controlled trial of methotrexate for patients with generalized myasthenia gravis. Neurology 2016;87(1):57-64

39 Díaz-Manera J, Martínez-Hernández E, Querol L, et al. Long-lasting treatment effect of rituximab in MuSK myasthenia. Neurology 2012;78(3):189-193

40 Nagappa M, Netravathi M, Taly AB, Sinha S, Bindu PS, Mahadevan A. Long-term efficacy and limitations of cyclophosphamide in myasthenia gravis. J Clin Neurosci 2014;21(11):1909-1914

41 Zhang Z, Yang C, Zhang L, Yi Q, Hao Z. Efficacy and safety of tacrolimus in myasthenia gravis: a systematic review and meta-analysis. Ann Indian Acad Neurol 2017;20(4):341-347 
42 Chen P, Feng H, Deng J, et al. Leflunomide treatment in corticosteroid-dependent myasthenia gravis: an open-label pilot study. J Neurol 2016;263(1):83-88

43 Cataneo AJM, Felisberto G, Cataneo DC Jr. Thymectomy in nonthymomatous myasthenia gravis - systematic review and meta-analysis. Orphanet J Rare Dis 2018;13(1):99
44 Kumar V, Kaminski HJ. Treatment of myasthenia gravis. Curr Neurol Neurosci Rep 2011;11(1):89-96

45 Jordan A, Freimer M. Recent advances in understanding and managing myasthenia gravis. F1000 Res 2018;7:7. 